\title{
Optical Properties of EVA Films Including V570 for Transparent Luminescent Solar Concentrator
}

\author{
Received November 25, 2019; revised December 31, 2019; accepted January 6, 2020
}

\author{
Kukhyun Jo, Seungyeon Hong, and Hyo Jung Kim* \\ Department of Organic Material Science and Engineering, Pusan National University, Busan 46241, Republic of Korea
}

*Corresponding author E-mail: hyojkim@pusan.ac.kr

\begin{abstract}
We fabricated a transparent luminescent solar concentrator (LSC) film using Lumogen F Violet 570 (V570) luminescent molecules and polyethylene-co-vinylaccetate (EVA) polymer. The transparent LSC was produced by a solution process (Doctor Blade coating) using a tetrahydrofuran solvent. We measured physical properties such as thickness, absorption efficiency, emission efficiency, and photocurrent characteristics of the LSC films. We also discuss the physical properties of the LSC films by observing the crystallinity and surface morphology using X-ray diffraction and atomic force microscopy. During the formation of LSC films, V570 molecules did not induce macroscopic changes, but had a significant effect on nanoscale morphology, i.e., surface roughness. In addition, changes in the light scattering characteristics were observed when the V570 concentration increased, which was found by X-ray diffraction to be related to the formation of V570 crystals, not EVA crystals. The light scattering and surface roughness of a film could be improved through heat treatment. As a result, we obtained $\sim 1.3$ times larger photocurrent in thermally treated LSC films.
\end{abstract}

Keywords: Lumogen F Violet 570, Luminescent solar concentrator, Transparent photovoltaic device, Concentration factor, Optical efficiency

\section{Introduction}

A luminescent solar concentrator (LSC) using luminescent molecules and a polymer matrix is a device for selective absorption of sunlight and for collecting the emitted light to the side edges of the collector through total internal reflection (TIR) [1-4]. An LSC reduces the amount of solar cells required for solar power generation $[5,6]$, and can selectively utilize the desired light in the solar spectrum according to the luminophore used. It is also possible to fabricate transparent photovoltaic devices by engineering the concentration of luminophores in LSC films. Thus, LSCs are attracting attention due to these many advantages $[7,8]$. However, the detailed principles of LSCs are complex because they involve a wide range of optical phenomena ranging from molecular physics such as light absorption and photoluminescence of luminophores, to macroscopic physics relating to light propagation inside the LSC via total internal reflection. Because an LSC concentrates light from photoluminescence of molecules, it is not straightforward to analyze the optical properties of LSC devices using conventional photovoltaic (PV).

The solar spectrum on the Earth's surface ranges from 280 to $4000 \mathrm{~nm}$, consisting of infrared (52.6\%), visible (43.0\%), and ultraviolet (4.6\%) light in the AM1.5G (Air Mass 1.5 Global) condition. The portion of UV is small, but it could induce material degradation in a PV device. Therefore, many technologies and materials have been developed to block UV for commercialization of high power PVs. Among various techniques, the conversion of UV light to longer wavelength light is considered a promising technique to improve both stability and energy efficiency in PV cells and modules [9].
Lumogen F Violet 570 (V570) is a 1,8-naphthalimide-based material that is widely used because of its unique optical properties and high quantum efficiency. It absorbs UV light up to $374 \mathrm{~nm}$ and emits visible range light at $427 \mathrm{~nm}$ [10-13]. Herein, we used V570 as a luminescent dye with polyethylene-co-vinylaccetate (EVA) polymer to produce a transparent LSC. We analyzed the relationship between the optical properties and structure of the transparent LSC film, and we attempted to understand the light collection performance for our fabrication conditions.

\section{Materials and methods}

\subsection{LSC preparation}

EVA polymer, V570 luminescent material, and tetrahydrofuran (THF) solvent were manufactured by LG chemicals (EP28015, Korea), BASF (Lumogen F Violet 570, Korea) and Reagents DUKSAN (No. 216 , Korea), respectively. A solution of $20 \mathrm{wt} \%$ EVA polymer in THF solvent was prepared, then V570 was added in proportions of $0.0,0.4$, $0.8,1.2$, and $1.6 \mathrm{wt} \%$ based on the mass of EVA before addition of solvent. The solution was stirred for $2 \mathrm{~h}(700 \mathrm{rpm})$ at $70{ }^{\circ} \mathrm{C}$ (set temperature of the hot plate).

The five solutions $(0.0,0.4,0.8,1.2$, and $1.6 \mathrm{wt} \%)$ were coated by Doctor Blade coater (MTI Korea, Korea). The gap between the blade edge and the substrate was $500 \mu \mathrm{m}$ during the coating process. Coated films were dried for $3 \mathrm{~min}$ at room temperature (RT), then transferred onto a hot plate for $10 \mathrm{~min}$ drying at $60^{\circ} \mathrm{C}$. Some of the LSC films were annealed at $150{ }^{\circ} \mathrm{C}$ for $10 \mathrm{~min}$ to observe the change of LSC 
properties caused by heat treatment. We measured thickness of the films at 5 points each to obtain an average thickness by Digimatic Micrometer (Mitutoyo, Japan).

\subsection{Optical properties}

The transmittance and quantum efficiency were measured using a UV-vis spectrophotometer (V-750, Jasco) and integrated sphere equipped spectrofluorometer (Quantaurus-QY c11347, Hamamatsu). Then, the absorption and emission efficiency of LSC were calculated by Eqs. (1)-(3).

Absorption Ef ficiency of LSC

$=\frac{\text { The intensity of absorbed solar irradiance at } L S C}{\text { The intensity of incident solar irradiance }}$

$$
\begin{gathered}
=\frac{\int_{\lambda_{1}}^{\lambda_{2}} I_{A M 1.5 G}(\lambda) \times A(\lambda) d \lambda}{\int_{280 n m}^{4000 n m} I_{A M 1.5 G}(\lambda) d \lambda} \\
A(\lambda)=T_{0}(\lambda)-T(\lambda)
\end{gathered}
$$

Emission Efficiency of LSC $=\frac{\text { The photoluminescence intensity emitted at } L S C}{\text { The intensity of absorbed solar irradiance at } L S C}$

$$
=\frac{\int_{\lambda_{1}}^{\lambda_{2}} I_{A M 1.5 G}(\lambda) \times A(\lambda) \times P L Q Y(\lambda) d \lambda}{\int_{\lambda_{1}}^{\lambda_{2}} I_{A M 1.5 G}(\lambda) \times A(\lambda) d \lambda}
$$

In Eq. (1), $I_{A M 1.5 G}(\lambda)$ and $A(\lambda)$ are the incident solar irradiation and absorption of the LSC, respectively. $T_{0}(\lambda)$ and $T(\lambda)$ in Eq. (2) are the transmittance of the pure EVA film and the LSC films. In Eq. (3), $\operatorname{PLQY}(\lambda)$ is the photoluminescence quantum yield of LSC. $\lambda_{1}$ and $\lambda_{2}$ are the wavelengths where the absorption of LSCs begins and ends. $\lambda_{1}$ and $\lambda_{2}$ are 300 and $410 \mathrm{~nm}$ for LSCs including V570, respectively.

\subsection{Photocurrent characterization}

We attached a c-Si solar cell to the LSC film samples to measure the photocurrent. An Oriel solar simulator (Newport, Class AAA solar simulator) was used to simulate the AM1.5G condition and the photoelectric properties were measured using a Keithley 4200 Source Measure Unit. The concentration factor was calculated by Eq. (4).

$$
\begin{gathered}
\text { The Concentration Factor }(C) \\
=\frac{\text { The intensity of light concentrated by the } L S C}{\text { The intensity of incident solar irradiance }} \\
=\frac{J_{s c}-J_{s c, 0}}{J_{s c, \text { cell }}}
\end{gathered}
$$

$J_{s c}$ and $J_{s c, 0}$ are the short-circuit current densities of the LSC element (EVA with luminescent material) and pure EVA film (without luminescent material), respectively, and $J_{s c, \text { cell }}$ is the photocurrent density when the c-silicone solar cell is directly irradiated under the AM1.5G condition. The value of $J_{\text {sc, cell }}$ was $40.94 \mathrm{~mA} / \mathrm{cm}^{2}$. The photocurrent $\left(J_{s c, 0}\right)$ was observed for the pure EVA film without the luminescent material for the calculation as described in Eq. (4).

\subsection{Crystal and surface structure}

We performed X-ray diffraction (XRD) (X'Pert 3, Malvern Panalytical) and atomic force microscopy (AFM) (NX10, Park Systems) on the film samples. For XRD measurement, we adopted the specular reflection mode with $1.54 \AA \mathrm{X}$-rays from a Cu target. AFM images were obtained by the non-contact mode $(\mathrm{NCM})$ in a $50 \times 50 \mu \mathrm{m}^{2}$ sized region.

\section{Results and discussion}

Figure 1 shows the variation of thickness of the LSC film with the concentration of V570, which shows that the thickness change of the films was not large by the increase of V570 concentration. In pristine films (without heat treatment), the average thickness and standard deviation was 62.3 and $1.94 \mu \mathrm{m}$. After the heat treatment at $150{ }^{\circ} \mathrm{C}$ for $10 \mathrm{~min}$, the film thickness increased by $2.4 \%$ to an average of $63.8 \mu \mathrm{m}$. This result implies that the addition of V570 to the EVA / THF solution did not induce a macroscopic change when the concentration of V570 was less than $1.6 \mathrm{wt} \%$.

The effects of treatment on the transmission are shown in Figs. 2(a) and 2(b), and the absorption efficiency is shown in Fig. 2(c). The

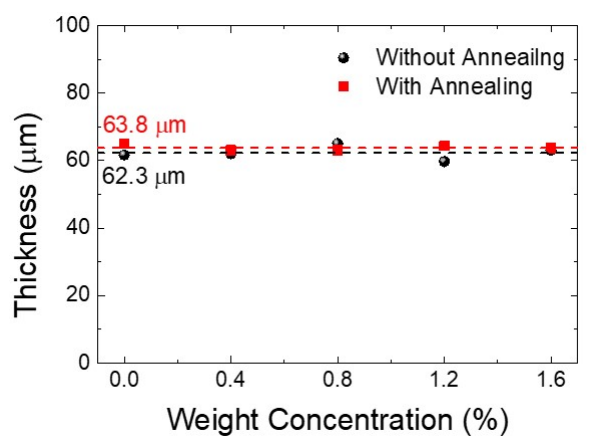

Figure 1. (Color online) Dependence of LSC film thickness on concentration of V570.

(a)

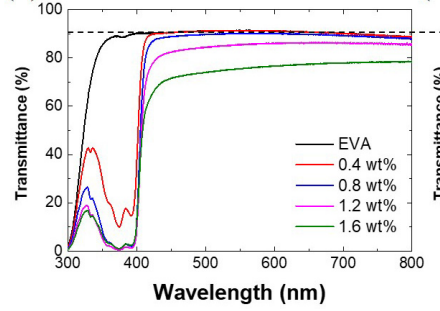

(b)

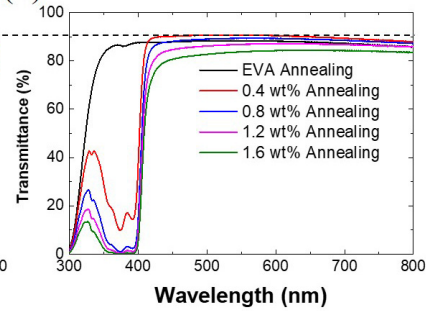

(c)

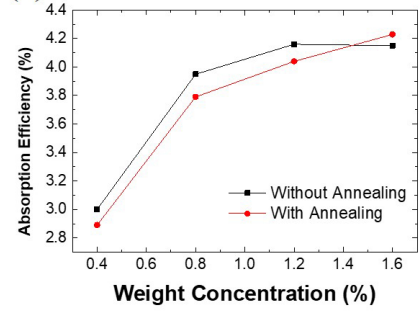

Figure 2. (Color online) Transmittance spectra of the LSC films (a) without heat treatment and (b) with heat treatment. (c) is the absorption efficiency of the LSC films variation with the concentration of $\mathrm{V} 570$. 
(a)

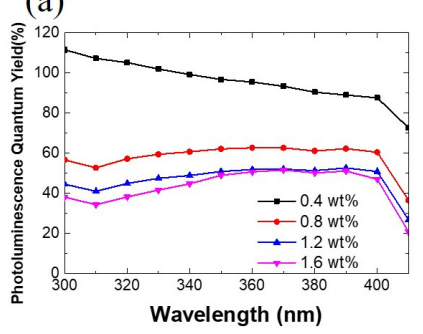

(b)

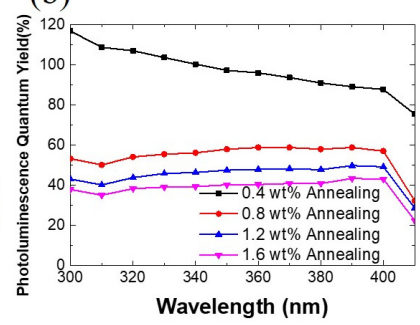

(c)

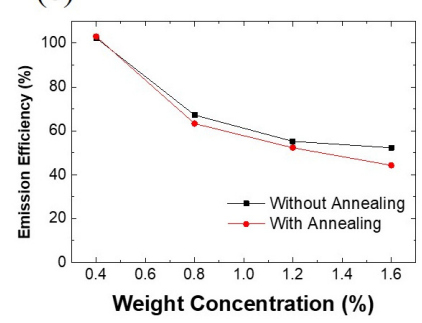

Figure 3. (Color online) Photoluminescence quantum yield (PLQY) of the LSC films (a) without heat treatment and (b) with heat treatment. (c) is the variation of the emission efficiency of the LSC films with the concentration of V570.

lowest transmittance was obtained at $374 \mathrm{~nm}$, which was not affected by either the heat treatment of the LSC film or the concentration of V570. In the $0.4 \mathrm{wt} \%$ film, the longest wavelength among the absorption peaks was $392 \mathrm{~nm}(3.16 \mathrm{eV})$, which is related to the change of the conjugated pattern of $\mathrm{p}$ electrons in the naphthalimide group in V570 [14]. Absorption peaks occurring at wavelengths of 374, 355, and 331 $\mathrm{nm}$, and lower than $392 \mathrm{~nm}$ were related to the coupling of the $392 \mathrm{~nm}$ absorption band and the molecular vibration mode. The transmittance decreased as the concentration of V570 increased in the longer wavelength region (> $400 \mathrm{~nm}$ ) where V570 does not absorb light. The decrease of transmittance at longer wavelength originated from the light scattering of LSC film, not the absorption characteristics of the V570 itself.

As shown in Fig. 2(b), heat treatment did not induce large changes of the transmittance spectra compared to pristine films. However, it is obvious that the heat-treated films exhibited decreased transmittance as the V570 concentration increased. For non-annealed films, the average visible transmittance (AVT) of EVA was $90.8 \%$. The AVT of LSC films was $89.8,87.8,82.7$, and $73.1 \%$ in $0.4 \mathrm{wt} \% .0 .8,1.2$, and $1.6 \mathrm{wt} \%$ films, respectively. After heat treatment, the AVT changed to 88.0 \% in EVA films. It was 89.3, 87.1, 84.0, and $80.5 \%$ in 0.4 wt\%. 0.8 , 1.2 , and $1.6 \mathrm{wt} \%$ heat treated films, respectively. In addition, the light scattering of the LSC film also decreased after heat treatment. Transmission of the pure EVA film slightly decreased after the heat treatment as shown in Figs. 2(a) and 2(b), and the absorption efficiency of LSC films from Eq. (4) decreased after heat treatment as shown in Fig. 2(c). The drop of transmission in pure EVA films was related to the increment of reflectance after heat treatment, which seems to be related to changes of surface morphology.

Figures 3(a) and 3(b) present the quantum efficiencies of LSC films in the wavelength ranges from 300 to $410 \mathrm{~nm}$. The highest quantum efficiency was observed at $0.4 \mathrm{wt} \%$ condition in both pure and heat-treated films. The $0.4 \mathrm{wt} \%$ film shows over $100 \%$ efficiency in the region below $340 \mathrm{~nm}$, which can be caused by the low reflectivity of the integrating sphere related to an inner coating problem
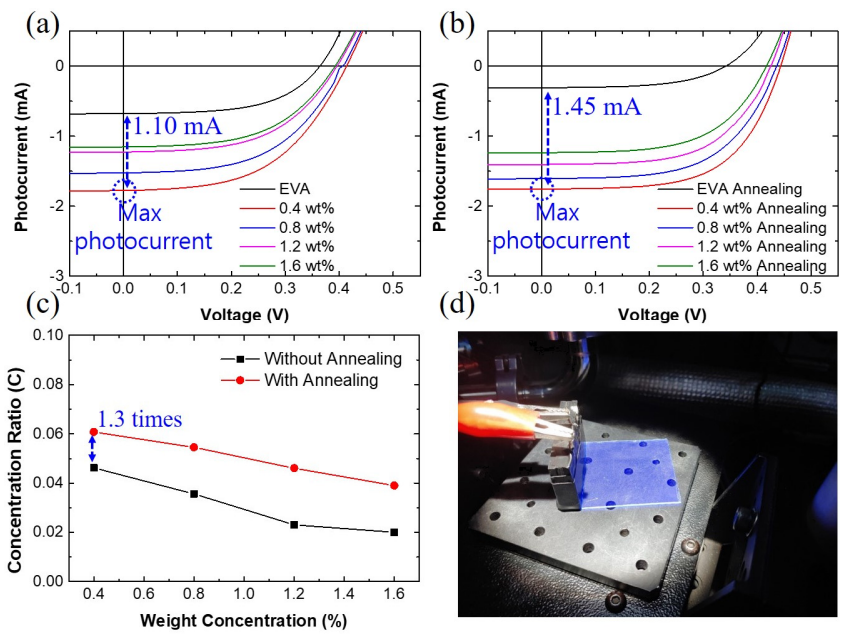

Figure 4. (Color online) Photocurrent properties of the LSC films depending on bias voltage (a) without heat treatment and (b) with heat treatment. (c) is the dependence of the concentration ratio of the LSC films with the concentration of V570. (d) shows the photograph of the photocurrent measurement device with the $0.4 \mathrm{wt} \%$ V 570 LSC attached to the c-Si solar cell.

[15]. For concentrations higher than $0.8 \mathrm{wt} \%$, the quantum efficiency of the LSC film slightly decreased after heat treatment compared to pristine films. Figure 3(c) shows the emission efficiency calculated using Eq. (3). As the V570 concentration increased, the emission efficiency decreased. The decrease of emission efficiency originated from the increase of intermolecular interactions, which resulted in nonradiative energy transfer.

We attached a c-Si solar cell to the film samples and measured the photocurrent, with the data shown in Figs. 4(a) and 4(b). As the concentration of V570 increased, the short-circuit current (the current flowing at zero voltage) decreased. In principle, pure EVA film should not have an LSC effect, and the photocurrent from pure EVA film should be zero. However, photocurrent was observed from EVA as shown in Figs. 4(a) and 4(b) due to small amount of light scattering in the EVA film. Therefore, we subtracted the photocurrent measured from pure EVA to evaluate the LSC effect of V570 concentration. The highest photocurrent was measured for $0.4 \mathrm{wt} \%$ films in both pristine and heat-treated films. The photocurrents (corrected for that from pure EVA film) from the $0.4 \mathrm{wt} \%$ sample were 1.10 and $1.45 \mathrm{~mA}$ for the pristine and heat treatment case, respectively. Figure 4(c) shows the light concentration ratio calculated using Eq. (4) for various concentrations of V570. The heat treatment of the film improved the total reflection, and as a result, the light concentration factor increased by $\sim 1.3$ times compared to pristine films. When the concentration of V570 increased, the concentration factor decreased because the emission efficiency in Fig. 2(c) significantly decreased even though the absorption efficiency increased. The concentration factor was reduced due to the low emission efficiency. Light scattering in the LSC film can induce reduction of the total internal reflection efficiency, and can also induce reduction of the concentration factor.

It is generally known that light scattering in polymer films is deeply related to crystallinity and surface properties of the film [16,17]. Therefore, we used XRD and AFM to understand the crystallinity and morphology at each sample preparation condition as shown in Figs. 5 and 6. The diffraction peak of Fig. 5 is from the alkyl chain of V570, and it indicates the formation of V570 crystals in the film, with a 
(a)

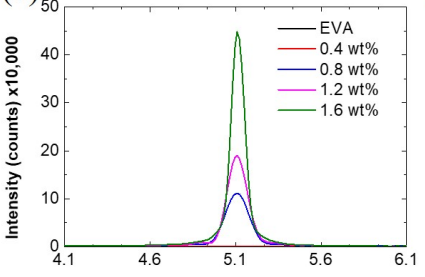

(c)

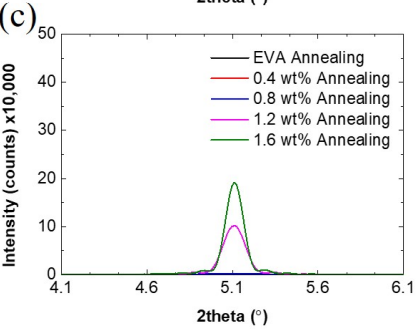

(b)

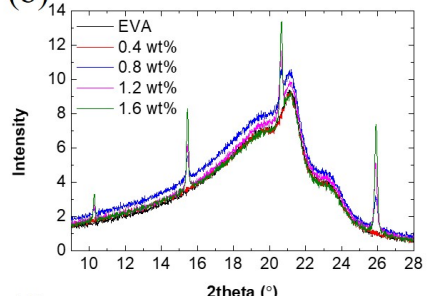

(d)

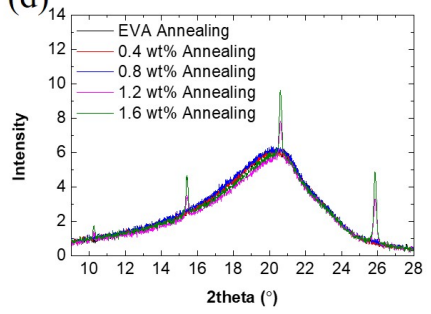

Figure 5. (Color online) (a) and (b) are XRD spectra of LSC films without heat treatment. (c) and (d) are XRD profile of LSC films with heat treatment. (a) and (c) show the diffraction peak from the alkyl chain of $\mathrm{V} 570$, while (b) and (d) show a broad $X R D$ pattern showing peaks related to $P E$.

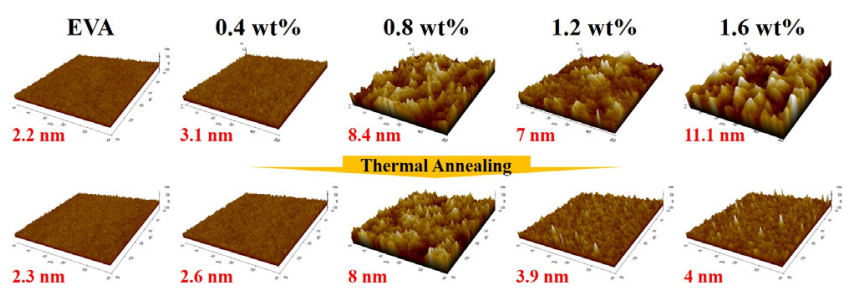

Figure 6. (Color online) Atomic force microscope images showing surface morphology of LSC films (top) without and (bottom) with heat treatment. (50 ×50 $\mu \mathrm{m}$ size)

magnitude that increases with increased concentration of V570. Above $0.8 \mathrm{wt} \%$, the V570 crystal peak was clearly observed, which is in good agreement with the changes of light scattering characteristics in Fig. 2 (a). Figures 5(b) and 5(d) show the peaks of PE (polyethylene) group for different concentrations of V570, and which imply that the crystallinity of PE was not changed by the concentration of V570. However, heat treatment caused a reduction of crystallinity of $\mathrm{PE}$ as shown in Fig. 5(d). Therefore, it seems that the light scattering was not related to PE in this system because the light scattering was dependent on both concentration and heat treatment. As shown in Fig. 5(c), the V570 peak decreased after heat treatment, which is in good agreement with the scattering reduction results in Fig. 2(b). The XRD results thus imply that the scattering characteristics of the LSC film are mainly caused by the crystals of V570, not the crystals of EVA.

After heat treatment, the formation of V570 crystals decreased, but the quantum efficiency of the LSC film did not increase, as shown in Fig. 2(b). This relationship was related to stronger interaction of the V570 molecules after the heat treatment. Based on these XRD and light scattering results, we could confirm that heat treatment reduced V570 crystal formation but did not interfere with aggregation to the extent that it destroys intermolecular interactions.

The surface roughness was modified by the addition of V570 and heat treatment as shown in Fig. 6. In Fig. 1, the addition of V570 did not induce macroscopic changes of the films. However, the nano-

scale roughness increased with addition of V570. At the condition of $0.4 \mathrm{wt} \%$, the surface roughness was similar to the pure EVA film. Heat treatment induced flattening of the surface, as shown in the AFM images. It seems that the role of heat treatment was to alleviate the aggregation of V570. The reduction of surface roughness could suppress the light scattering of the LSC film along with the reduction in the V570 crystals. In addition, we expect that the flattening of the surface increased the light collection ratio because a smooth surface has improved total internal reflection.

\section{Conclusions}

We fabricated transparent LSC samples using light-emitting molecules that absorbed light in the UV region, and analyzed their optical properties. We found that light scattering in the LSC films was mainly dependent on the crystallinity of V570 rather than the PE group of EVA matrix. Heat treatment did not increase the absorption and emission efficiency of the LSC film, but it reduced both surface roughness and light scattering. As a result, heat treatment could contribute to much improved light collection performance in LSC films based on V570 and EVA.

\section{Acknowledgements}

This work was supported by a 2-year Research Grant of Pusan National University, and was also supported by the BK21+ project in 2019.

\section{References}

[1] G. Marianetti, M. Lessi, V. Barone, F. Bellina, A. Pucci, and P. Minei, Dyes Pigm. 157, 334 (2018).

[2] H. Zhao, D. Benetti, X. Tong, H. Zhang, Y. Zhou, G. Liu, D. Ma, S. Sun, Z. M. Wang, Y. Wang, and F. Rosei, Nano Energy 50, 756 (2018).

[3] S. Chandra, M. Rafiee, J. Doran, and S. J. Mc Cormack, Sol. Energ. Mat. Sol. C. 182, 331 (2018).

[4] C. Papucci, T. A. Geervliet, D. Franchi, O. Bettucci, A. Mordini, G. Reginato, F. Picchioni, A. Pucci, M. Calamante, and L. Zani, Eur. J. Org. Chem. 20-21, 2657 (2018).

[5] S. Y. Hong, S. Lee, and H. J. Kim, Appl. Sci. Converg. Technol. 28, 1 (2019).

[6] A. P. Jadhav, S. Khan, S. J. Kim, and S. H. Cho, Appl. Sci. Converg. Technol. 23, 221 (2014).

[7] M. R. Bergren, N. S. Makarov, K. Ramasamy, A. Jackson, R. Guglielmetti, and H. McDaniel, ACS Energy Lett. 3, 520 (2018).

[8] B. Zhang, J. L. Banal, D. J. Jones, B. Z. Tang, K. P. Ghiggino, and W. W. Wong, Mater. Chem. Front. 2, 615 (2018).

[9] F. Bella, G. Griffini, M. Gerosa, S. Turri, and R. Bongiovanni, J. Power Sources 283, 195 (2015).

[10] R. M. Duke, E. B. Veale, F. M. Pfeffer, P. E. Kruger, and T. Gunnlaugsson, Chem. Soc. Rev. 39, 3936 (2010).

[11] D. Alonso-Álvarez, D. Ross, E. Klampaftis, K. R. McIntosh, S. Jia, P. Storiz, T. Stolz, and B. S. Richards, Prog. Photovoltaics: Res. Appl. 23, 479 (2015).

[12] H. Ulla, B. Garudachari, M. N. Satyanarayan, G. Umesh, and A. M. Isloor, Opt. Mater. 36, 704 (2014).

[13] D. Kolosov, V. Adamovich, P. Djurovich, M. E. Thompson, and 
C. Adachi, J. Am. Chem. Soc. 124, 9945 (2002).

[14] D. Jacquemin, E. A. Perpète, G. Scalmani, M. J. Frisch, I. Ciofini, and C. Adamo, Chem. Phys. Lett. 448, 3 (2007).

[15] J. Gene, S. N. Park, H. P. Kim, and S. Park, Metrologia (2019).
[16] F. C. Stehling, C. S. Speed, and L. Westerman, Macromolecules 14, 698 (1981)

[17] E. Andreassen, Å. Larsen, K. Nord-Varhaug, M. Skar, and H. Øysæd, Polym. Eng. Sci. 42, 1082 (2002). 